\title{
Heavy bottom squark mass in the light gluino and light bottom squark scenario ${ }^{1}$
}

\author{
Zumin Luo ${ }^{2}$ and Jonathan L. Rosner ${ }^{3}$ \\ Enrico Fermi Institute and Department of Physics \\ University of Chicago, 5640 S. Ellis Avenue, Chicago, IL 60637
}

\begin{abstract}
Restrictive upper bounds on the heavy bottom squark mass when the gluino and one bottom squark are both light are based on the predicted reduction of $R_{b}$ (the fraction of $Z$ hadronic decays to $b \bar{b}$ pairs) in such a scenario. These bounds are found to be relaxed by the process $Z \rightarrow$ $b \overline{\tilde{b}} \tilde{g} / \bar{b} \tilde{b} \tilde{g}$, which may partially compensate for the reduction of $R_{b}$. The relaxation of bounds on the top squark and the scale-dependence of the strong coupling constant are also discussed.
\end{abstract}

PACS Categories: 11.30.Pb, 12.60.Jv, 13.38.Dg, 14.80.Ly

As a promising candidate for physics beyond the Standard Model (SM), the minimal supersymmetric standard model (MSSM) has been intensively studied in the past years. Recently there has been some interest $[1,2,3,4,5,7,8,9,10]$ in a MSSM scenario with a light gluino and a light bottom squark. It was first proposed by Berger et al. [1] to explain the excess of the cross section for bottom quark production at hadron colliders. In this scenario, the gluino has mass $m_{\tilde{g}}=12-16 \mathrm{GeV}$ and the light bottom squark has mass $m_{\tilde{b}}=2-5.5 \mathrm{GeV}$. The other bottom squark $\tilde{b}^{\prime}$, an orthogonal mixture of $\tilde{b}_{L}$ and $\tilde{b}_{R}$ to the light bottom squark, is assumed to be heavy, and so are all other supersymmetric (SUSY) particles. We follow the convention in Ref. [1] to define

$$
\left(\begin{array}{c}
\tilde{b} \\
\tilde{b}^{\prime}
\end{array}\right)=\left(\begin{array}{cc}
\cos \theta_{\tilde{b}} & \sin \theta_{\tilde{b}} \\
-\sin \theta_{\tilde{b}} & \cos \theta_{\tilde{b}}
\end{array}\right)\left(\begin{array}{c}
\tilde{b}_{R} \\
\tilde{b}_{L}
\end{array}\right) .
$$

In this scenario the $Z$ decay width $\Gamma_{Z}$ is reduced by SUSY-QCD corrections to the $Z b \bar{b}$ vertex [3]. However, some new decay processes contribute positively to $\Gamma_{Z}$ and may compensate for the reduction. Apart from $Z \rightarrow \tilde{g} \tilde{g}$, which was studied in Ref. [10] and found to have a decay width of order $0.1 \mathrm{MeV}$, there exist two more important decays, $Z \rightarrow b \overline{\tilde{b}} \tilde{g} / \bar{b} \tilde{b} \tilde{g}$ and $Z \rightarrow q \bar{q} \tilde{g} \tilde{g}$. The first process is $\sim \alpha \alpha_{s}$ at the tree level and has a decay width of $1.9-5.9 \mathrm{MeV}$ depending on the sign of $\sin 2 \theta_{\tilde{b}}$ and the mass of the gluino [7]. The second process is $\sim \alpha \alpha_{s}^{2}$ and its decay width is calculated in a model-independent way to be $0.75-0.21 \mathrm{MeV}$ for $m_{\tilde{g}}=12-16 \mathrm{GeV}$ [6]. Additional "sbottom splitting" diagrams [9] raise $\Gamma(Z \rightarrow q \bar{q} \tilde{g} \tilde{g})$ by about $0.01 \mathrm{MeV}$.

By studying the SUSY-QCD effects on $R_{b}$, Cao et al. [3] showed that the mass of the heavy bottom squark should not exceed $195 \mathrm{GeV}$ at the $3 \sigma$ level. Taking

\footnotetext{
${ }^{1}$ Enrico Fermi Institute preprint EFI 03-27, hep-ph/0306022, to be submitted to Phys. Lett. B.

zuminluo@midway.uchicago.edu

${ }^{3}$ rosner@hep.uchicago.edu
} 
into account additional electroweak corrections to the gauge boson propagators and including other relevant $Z$-pole observables and the $W$-boson mass, Cho [4] found that the mass of $\tilde{b}^{\prime}$ is further constrained $(\leq 180 \mathrm{GeV})$ and that one of the top squarks $(\tilde{t})$ should be lighter than $98 \mathrm{GeV}$ at the $5 \sigma$ level. However, both of these analyses ignored the impact of the new $Z$ decays on the electroweak observables. Most notably, it was shown in Ref. [6] that $Z \rightarrow b \overline{\tilde{b}} \tilde{g} / \bar{b} \tilde{b} \tilde{g}$ may affect the measurement of $R_{b}$. Indeed, since the gluino is assumed to decay promptly to a bottom quark and a bottom squark, the final state in $Z \rightarrow b \overline{\tilde{b}} \tilde{g} / \bar{b} \tilde{b} \tilde{g}$ will be an energetic bottom jet backto-back with a "fat" jet consisting of a bottom quark and two light bottom squarks. The energetic bottom jet can add to $R_{b}$ if the analysis consists merely of counting $b$ candidates. Moreover, if the light bottom squark cannot escape the detector, the "fat" jet can also be tagged as a $b$ jet and contribute to $R_{b}[6,11]$. However, since the branching ratio of $Z \rightarrow b \overline{\tilde{b}} \tilde{g} / \bar{b} \tilde{b} \tilde{g}$ is only of order $10^{-3}$, current LEP data are not likely to be sensitive to it [11] without a dedicated search. Despite this, the $Z \rightarrow b \overline{\tilde{b}} \tilde{g} / \bar{b} \tilde{b} \tilde{g}$ decay plays a nonnegligible role in constraining the heavy bottom squark mass. In this Letter we argue that the upper bound on $m_{\tilde{b}^{\prime}}$ will be relaxed if this process can contribute a certain amount to the measured $\Gamma(Z \rightarrow b \bar{b})$.

The proposed scenario implicitly requires that $R$-parity be violated in bottom squark decays [4]. Assuming pure lepton-number violation in top squark decays $(\mathcal{B}(\tilde{t} \rightarrow \tau b)=1)$, the CDF Collaboration [12] has recently set a $95 \%$ confidence level lower limit at $122 \mathrm{GeV}$ on the light top squark mass $m_{\tilde{t}}$ in the framework of $R$-parity violating MSSM. We will show that the upper bound on $m_{\tilde{t}}$ can be raised above $122 \mathrm{GeV}$. However, we point out that the CDF lower limit is somewhat irrelevant to the proposed scenario. The top squark decay in the light gluino and light bottom squark scenario is not necessarily $R$-parity violating or purely lepton-numberviolating. An $R$-parity-violating decay invoked for the bottom squark does not tell us anything about how the top squark decays since the two processes arise from different terms in the Lagrangian.

The $Z b \bar{b}$ vertex in the Standard Model can be written as

$$
V^{\mu}(Z b \bar{b})=-i \frac{g}{2 \cos \theta_{W}}\left[\gamma^{\mu}\left(g_{V}^{b}-g_{A}^{b} \gamma^{5}\right)\right],
$$

where $g_{V}^{b}=-1 / 2+2 / 3 \sin ^{2} \theta_{W}, g_{A}^{b}=-1 / 2$, and $\theta_{W}$ is the weak angle. The SUSYQCD corrections to $g_{V}^{b}$ and $g_{A}^{b}$ have been calculated in the MSSM by a number of authors [3, 5, 13, 14, 15]. Using the analytical expressions in Ref. [14], we reproduce the numerical results in Ref. [3]. However, we cannot reproduce the numerical results in Ref. [5], which also disagree with the other references analytically.

For numerical evaluation, we take $\alpha_{s}\left(M_{Z}\right)=0.120, \sin ^{2} \theta_{W}=0.2311, m_{b}=4.1$ $\mathrm{GeV}, m_{\tilde{b}}=5.5 \mathrm{GeV}, m_{\tilde{g}}=16 \mathrm{GeV}, R_{b}^{\mathrm{SM}}=0.21569$ and $\Gamma_{Z}^{\mathrm{SM}}(\mathrm{had})=1.7429 \mathrm{GeV}[16]$. The greatest possible values are chosen for $m_{\tilde{b}}$ and $m_{\tilde{g}}$ so that the upper limit on $m_{\tilde{b}^{\prime}}$ is the least restrictive [3]. The change in $R_{b}$ with respect to its SM value is plotted in Fig. 1. For now we do not consider the possible effects of the new $Z$ decay processes. The bottom squark mixing angle $\theta_{\tilde{b}}$ is chosen to satisfy $\left|\sin \theta_{\tilde{b}}\right| \simeq \sqrt{2 \sin ^{2} \theta_{W} / 3} \simeq 0.39$ so that the $Z \tilde{b} \tilde{\tilde{b}}$ coupling is suppressed [1]. We allow $\left|\sin \theta_{\tilde{b}}\right|$ to vary between 0.30 and 
Table I: Upper bounds on the heavy bottom squark mass at the $3 \sigma$ level. $x$ is the contribution (in units of MeV) of the decays $Z \rightarrow b \bar{b} \tilde{g} / \bar{b} \tilde{b} \tilde{g}$ to measured $\Gamma(Z \rightarrow b \bar{b})$ as a result of mistagging of the "fat" jets.

\begin{tabular}{cc}
\hline \hline$x$ & Upper bound on $m_{\tilde{b}^{\prime}}(\mathrm{GeV})$ \\
\hline 1 & 215 \\
1.5 & 275 \\
2 & 365 \\
2.5 & 490 \\
3 & 655 \\
\hline \hline
\end{tabular}

0.45. The partial decay width $\Gamma(Z \rightarrow \tilde{b} \tilde{\tilde{b}})$ is about $(1.0,0.6) \mathrm{MeV}$ for $\left|\sin \theta_{\tilde{b}}\right|=$ $(0.30,0.45)$. Fig. 1 shows that the heavy bottom squark mass $m_{\tilde{b}^{\prime}}$ must be less than about $200 \mathrm{GeV}$ for $R_{b}$ to be within the $3 \sigma$ bound $\left(\delta R_{b} \geq-0.0013\right)$ set by the LEP experimental value [16].

Now suppose that a portion of the $Z \rightarrow b \bar{b} \tilde{g} / \bar{b} \tilde{b} \tilde{g}$ events cannot be distinguished from the $Z \rightarrow b \bar{b}$ ones and hence contribute $x \mathrm{MeV}$ to $\Gamma(Z \rightarrow b \bar{b})$. We shall take $1.0 \leq x \leq 3.0$. Note that the decay width for $Z \rightarrow b \overline{\tilde{b}} \tilde{g} / \bar{b} \tilde{b} \tilde{g}$ is approximately $4.4 \mathrm{MeV}$ for $m_{\tilde{b}}=3 \mathrm{GeV}, m_{\tilde{g}}=16 \mathrm{GeV}$ and $\sin \theta_{\tilde{b}} \simeq 0.39$ [7]. Since the two bottom quarks in the final states will hadronize in the detector, the hadronic decay width $\Gamma_{Z}$ (had) will also be affected. Furthermore, the final-state bottom squark can also contribute to the hadronic decay width via $R$-parity-violating decays to light quarks [1]. (Possibilities of a long-lived $\tilde{b}$ and lepton-number-violating decays of $\tilde{b}$ to hard leptons are both disfavored; see Refs. [1, 17].) Considering also other decays such as $Z \rightarrow \tilde{b} \tilde{b}, Z \rightarrow \tilde{g} \tilde{g}$ and $Z \rightarrow q \bar{q} \tilde{g} \tilde{g}$, we estimate that the total change in $\Gamma_{Z}(\mathrm{had})$ is $4.0 \mathrm{MeV}+\delta \Gamma(Z \rightarrow b \bar{b})$. Here $\delta \Gamma(Z \rightarrow b \bar{b})$ denotes the change in $\Gamma(Z \rightarrow b \bar{b})$ due to SUSY-QCD effects, as discussed above. The upper bound on $m_{\tilde{b}^{\prime}}$ can then be relaxed if the additional decays contribute positively to $R_{b}$, i.e., $\left[\Gamma^{\mathrm{SM}}(Z \rightarrow b \bar{b})+x\right] /\left[\Gamma_{Z}^{\mathrm{SM}}(\mathrm{had})+4.0\right]>R_{b}^{\mathrm{SM}}$, or equivalently $x / 4.0>R_{b}^{\mathrm{SM}}$. For example, the new upper bound on $m_{\tilde{b}^{\prime}}$ is around $275 \mathrm{GeV}$ at the $3 \sigma$ level for $x=1.5$; see Fig. 2, where we take into account the additional $Z$ decays and plot $\delta R_{b}$ as a function of $m_{\tilde{b}^{\prime}}$ for $m_{\tilde{b}}=5.5 \mathrm{GeV}, m_{\tilde{g}}=16$ $\mathrm{GeV}, \sin \theta_{\tilde{b}}=0.30$ and $x=1.0-3.0$. The new upper bounds at the $3 \sigma$ level are presented in Table I for various values of $x$.

SUSY-QCD corrections to $g_{V}^{b}$ and $g_{A}^{b}$ also affect some other related $Z$-pole electroweak observables. These are $\Gamma_{Z}, \Gamma(\mathrm{had}), R_{\ell}, R_{c}, A_{\mathrm{FB}}^{(0, b)}$ and $A_{b}$, where we use the notations in Ref. [16]. Some or all of these observables can also be affected by the additional $Z$ decay processes in the proposed scenario. We show in Table II the comparison of the predictions in the SM and in the proposed scenario for $m_{\tilde{b}^{\prime}}=180,275$ $\mathrm{GeV}$, with or without the additional decays taken into account. (Little can be known of the effect of those decays on $A_{\mathrm{FB}}^{(0, b)}$ and $A_{b}$.) One can see that raising $m_{\tilde{b}^{\prime}}$ from 180 $\mathrm{GeV}$ to $275 \mathrm{GeV}$ does not make the predictions agree much worse with experiments, especially after the additional decays are considered. Thus, the constraint on $R_{b}$ plays 


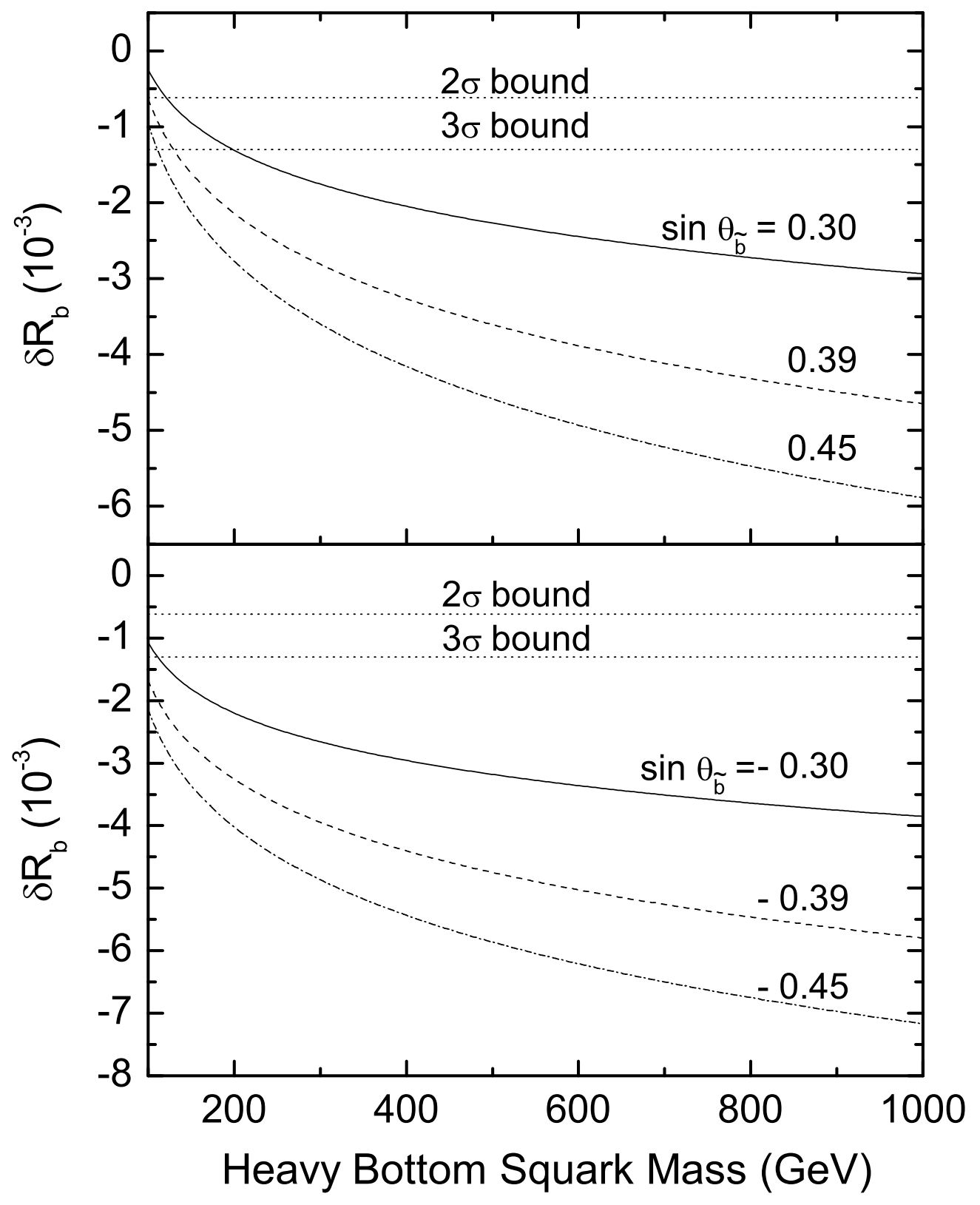

Figure 1: Change in $R_{b}$ due to SUSY-QCD effects alone in the light gluino and light bottom squark scenario with respect to its Standard Model value as a function of the heavy bottom squark mass. Here we take $m_{\tilde{b}}=5.5 \mathrm{GeV}, m_{\tilde{g}}=16 \mathrm{GeV}$. The horizontal dotted lines correspond to $2 \sigma$ and $3 \sigma$ bounds set by the LEP $R_{b}$ measurements [16]. 


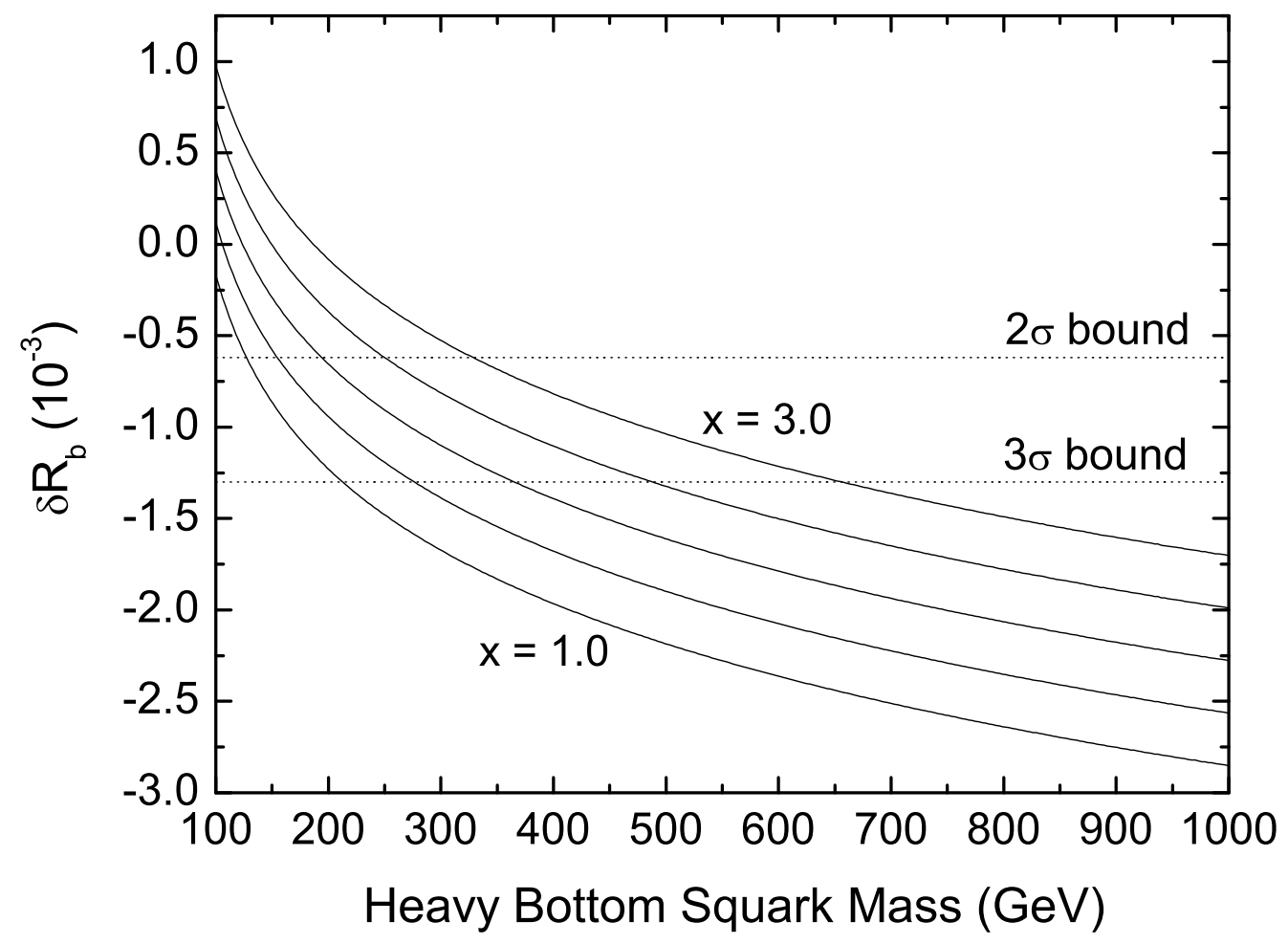

Figure 2: Change in $R_{b}$ in the light gluino and light bottom squark scenario with respect to its Standard Model value as a function of the heavy bottom squark mass. SUSY-QCD effects and possible impact of the decay process $Z \rightarrow b \bar{b} \tilde{g} / / \bar{b} \tilde{b} \tilde{g}$ are combined. $x$ is the contribution (in units of $\mathrm{MeV}$ ) of the process to measured $\Gamma(Z \rightarrow b \bar{b})$ as a result of mistagging of the "fat" jets. From bottom to top, $\mathrm{x}$ takes the values $1.0,1.5,2.0,2.5$ and 3.0. Here we use $m_{\tilde{b}}=5.5 \mathrm{GeV}, m_{\tilde{g}}=16 \mathrm{GeV}$ and $\sin \theta_{\tilde{b}}=0.30$. The horizontal dotted lines correspond to $2 \sigma$ and $3 \sigma$ bounds set by the LEP $R_{b}$ measurements [16]. 
Table II: Deviations of the predictions for some $Z$-pole observables from experimental values [16]. $\mathrm{MSSM}_{180,275}(1)[(2)]$ denotes the prediction in the light gluino and light bottom squark scenario for $m_{\tilde{b}^{\prime}}=180,275 \mathrm{GeV}$ before [after] taking into account the additional $Z$ decays. Here we use $m_{\tilde{b}}=5.5 \mathrm{GeV}, m_{\tilde{g}}=16 \mathrm{GeV}, \sin \theta_{\tilde{b}}=0.30$.

\begin{tabular}{cccccc}
\hline \hline Observable & SM & MSSM $_{180}(1)$ & MSSM $_{180}(2)$ & MSSM $_{275}(1)$ & MSSM $_{275}(2)$ \\
\hline$\Gamma_{Z}$ & $0.61 \sigma$ & $-0.52 \sigma$ & $1.87 \sigma$ & $-1.00 \sigma$ & $1.39 \sigma$ \\
$\Gamma($ had $)$ & $-0.75 \sigma$ & $-2.05 \sigma$ & $-0.05 \sigma$ & $-2.60 \sigma$ & $-0.60 \sigma$ \\
$R_{\ell}$ & $-1.20 \sigma$ & $-1.82 \sigma$ & $-0.87 \sigma$ & $-2.08 \sigma$ & $-1.12 \sigma$ \\
$R_{c}$ & $-0.19 \sigma$ & $-0.11 \sigma$ & $-0.23 \sigma$ & $-0.07 \sigma$ & $-0.20 \sigma$ \\
$A_{\mathrm{FB}}^{(0, b)}$ & $3.18 \sigma$ & $3.39 \sigma$ & - & $3.43 \sigma$ & - \\
$A_{b}$ & $0.69 \sigma$ & $0.85 \sigma$ & - & $0.88 \sigma$ & - \\
\hline \hline
\end{tabular}

the most important role in setting an upper bound on $m_{\tilde{b}^{\prime}}$, which we have already shown can be relaxed to about $275 \mathrm{GeV}$ if the process $Z \rightarrow b \overline{\tilde{b}} \tilde{g} / \bar{b} \tilde{b} \tilde{g}$ contributes 1.5 $\mathrm{MeV}$ (about one third of its own decay width) to the measured $\Gamma(Z \rightarrow b \bar{b})$. With $m_{\tilde{b}^{\prime}} \simeq 275 \mathrm{GeV}$, we argue below that the upper bound on $m_{\tilde{t}}$ can also be relaxed.

The constraint on $m_{\tilde{t}}$ originates primarily from $S U(2)_{L}$ gauge symmetry and the constraint on oblique corrections, i.e., electroweak corrections to gauge boson propagators. These corrections are parametrized by $\Delta S_{Z}, \Delta T_{Z}$ and $\Delta M_{W}$ in Ref. [15]. It has been shown $[4,15]$ that the dominant contributions to these parameters come from the bottom and top squark corrections (denoted by $\Delta T$ ) to the $T$-parameter [18]. Since $T$ is defined in terms of vacuum polarization amplitudes of the $S U(2)_{L}$ gauge bosons, only the left-handed components of the squarks can contribute to $\Delta T$. For given bottom squark masses $m_{\tilde{b}}, m_{\tilde{b}^{\prime}}$ and left-right $(L-R)$ mixing angle $\theta_{\tilde{b}}$, large $L-R$ mixing for the top squarks is favored so that the left-handed component of the lighter top squark mass eigenstate is relatively suppressed and therefore a small $\Delta T$ can be obtained [4]. The $L-R$ mixing for the top squarks is parametrized by $A_{\text {eff }}^{t}$ in Ref. [4], where it is constrained to be no less than $300 \mathrm{GeV}$ for $m_{\tilde{b}^{\prime}}=180 \mathrm{GeV}$ in the light gluino and light bottom squark scenario. This leads to a very restrictive upper bound $(98 \mathrm{GeV})$ on $m_{\tilde{t}}$. For $A_{\text {eff }}^{t}=300 \mathrm{GeV}$ and a consistent set of masses and mixing angles $\left(m_{\tilde{b}}=5.5 \mathrm{GeV}, m_{\tilde{b}^{\prime}}=180 \mathrm{GeV}, m_{\tilde{t}}=98 \mathrm{GeV}, m_{\tilde{t}^{\prime}}=340 \mathrm{GeV}\right.$, $\left.\theta_{\tilde{b}}=\arcsin 0.30 \simeq 17.5^{\circ}, \theta_{\tilde{t}}=49.2^{\circ}\right)$, we find $\Delta T \simeq 0.081$. Larger values for $\Delta T$ are obtained if $m_{\tilde{t}}>98 \mathrm{GeV}$. Without going through a similar $\chi^{2}$ fit procedure to that in Ref. [4], we regard 0.08 as a threshold value for $\Delta T$ below which oblique corrections are assumed to be acceptably small. For the same $A_{\text {eff }}^{t}$ but with $m_{\tilde{b}^{\prime}}$ raised to $275 \mathrm{GeV}$, we find that smaller values for $\Delta T$ can be obtained for $m_{\tilde{t}}$ as large as 210 $\mathrm{GeV}$; see Table III. This implies that the upper bound on $m_{\tilde{t}}$ may be considerably relaxed if the process $Z \rightarrow b \tilde{b} \tilde{g} / \bar{b} \tilde{b} \tilde{g}$ can contribute a certain amount to the measured $\Gamma(Z \rightarrow b \bar{b})$.

One can even tolerate much larger values of $\Delta T$ if one is prepared to consider heavier Higgs boson masses and small positive changes in $S$ in precision electroweak 
Table III: Examples for $m_{\tilde{t}}>98 \mathrm{GeV}$ and $\Delta T<0.08$. We take $m_{\tilde{b}}=5.5 \mathrm{GeV}$, $m_{\tilde{b}^{\prime}}=275 \mathrm{GeV}, \sin \theta_{\tilde{b}}=0.30$.

\begin{tabular}{cccc}
\hline \hline$m_{\tilde{t}}(\mathrm{GeV})$ & $m_{\tilde{t}^{\prime}}(\mathrm{GeV})$ & $\theta_{\tilde{t}}$ & $\Delta T$ \\
\hline 100 & 357 & $31.9^{\circ}$ & 0.061 \\
165 & 368 & $38.1^{\circ}$ & 0.070 \\
210 & 387 & $46.4^{\circ}$ & 0.079 \\
\hline \hline
\end{tabular}

fits $[19,20]$. In one example [20], by letting the Higgs boson mass rise to about $1 \mathrm{TeV}$, one can accommodate $\Delta T$ as large as 0.5 at the price of $\Delta S=0.3$ while retaining an acceptable fit to the data.

Finally we comment briefly on the scale-dependence ("running") of the strong coupling constant $\alpha_{s}$ in the light gluino and light bottom squark scenario. In the context of this scenario, we showed in a previous work [8] that $\alpha_{s}\left(M_{Z}\right)$ fell in the range $(0.130-0.135) \pm(>) 0.003$ if extrapolated from low mass scales (such as $\left.m_{b}\right)$ and in the range $(0.123-0.131) \pm 0.005$ if extracted directly from the $Z$-pole observable $\Gamma_{Z}(\mathrm{had})$. These two ranges overlap with one another and no clear-cut decision could be made in favor of either the Standard Model or the proposed SUSY scenario. However, we were only able to evaluate $\Gamma(Z \rightarrow \tilde{g} \tilde{g})$ very roughly and did not take into account the decay process $Z \rightarrow b \bar{b} \tilde{g} / \bar{b} \tilde{b} \tilde{g}$ at that time. With both of these processes now well understood and considered, the possible range of the extracted $\alpha_{s}\left(M_{Z}\right)$ from $\Gamma_{Z}($ had $)$ turns out to be different. For numerical purposes, we again use $\alpha_{s}\left(M_{Z}\right)=0.120$, $m_{\tilde{b}}=5.5 \mathrm{GeV}, m_{\tilde{g}}=16 \mathrm{GeV}$, $\sin \theta_{\tilde{b}}=0.30$ and take $120 \mathrm{GeV} \leq m_{\tilde{b}^{\prime}} \leq 655 \mathrm{GeV}$. Here the lower bound on $m_{\tilde{b}^{\prime}}$ is based on a recent analysis by Berger et al. [21]. The SUSY-QCD correction to $\Gamma(Z \rightarrow b \bar{b})$ is then $\delta \Gamma(Z \rightarrow b \bar{b})=-(1.3-5.6) \mathrm{MeV}$. We neglect the relatively small changes in $\delta \Gamma(Z \rightarrow b \bar{b})$ due to different choices of $\alpha_{s}\left(M_{Z}\right)$. Assuming the contribution of the new decay processes to $\Gamma_{Z}(\mathrm{had})$ is $4 \mathrm{MeV}$, the net change in the predicted $\Gamma_{Z}$ (had) is then $(-1.6-+2.7) \mathrm{MeV}$. This change can be accounted for by tuning $\alpha_{s}\left(M_{Z}\right)$ to lie in the range $(0.118-0.126) \pm 0.005$. It is lower than the extrapolated range from low mass scales, but not at a statistically significant level. The heavy sbottom mass should be better constrained to reduce the indeterminacy.

To summarize, we have shown that it is possible to circumvent restrictive upper bounds that have been placed on the heavy bottom squark mass when the gluino and one bottom squark are both light. The reduction of $R_{b}$ predicted in such treatments, which would conflict with data, can be compensated by a contribution to the $b$ quark production cross section from the process $Z \rightarrow b \overline{\tilde{b}} \tilde{g} / \bar{b} \tilde{b} \tilde{g}$. In such a case one expects a fast $b$ quark jet to be accompanied by a "fat jet" associated with the hadronization of the $\tilde{b} \tilde{g}$ or $\tilde{b} \tilde{g}$ system. The relaxation of the bounds on the heavy bottom squark mass is accompanied by a corresponding relaxation of bounds on the top squark mass. The situation of the scale-dependence of the strong coupling constant $\alpha_{s}$ becomes less favorable to the light gluino and light bottom squark scenario after the decay process 
$Z \rightarrow b \bar{b} \tilde{g} / \bar{b} \tilde{b} \tilde{g}$ is taken into account. However, improved understanding of the effects of the new decay processes in the scenario on $R_{b}$ and $\Gamma_{Z}(\mathrm{had})$ is needed for a clear-cut conclusion.

\section{Acknowledgements}

We are grateful to S. Baek, E. L. Berger, J. Cao and R. Hawkings for helpful discussions. We would also like to thank D. Rainwater and T. M. Tait for useful comments. This work was supported in part by the U. S. Department of Energy through Grant Nos. DE-FG02-90ER-40560.

\section{References}

[1] E. L. Berger, B. W. Harris, D. E. Kaplan, Z. Sullivan, T. M. P. Tait and C. E. M. Wagner, Phys. Rev. Lett. 86 (2001) 4231; E. L. Berger, arXiv:hepph/0103145, published in BCP4: International Workshop on B Physics and CP Violation, Ise-Shima, Japan, 19-23 February 2001, edited by T. Ohshima and A. I. Sanda (World Scientific, Singapore, 2001), p. 221.

[2] A. Dedes and H. K. Dreiner, JHEP 0106 (2001) 006; E. L. Berger and L. Clavelli, Phys. Lett. B 512 (2001) 115; A. K. Leibovich and D. Rainwater, Phys. Rev. Lett. 88 (2002) 221801; E. L. Berger and J. Lee, Phys. Rev. D 65 (2002) 114003; T. Becher, S. Braig, M. Neubert and A. L. Kagan, Phys. Lett. B 540 (2002) 278; E. L. Berger, C. W. Chiang, J. Jiang, T. M. Tait and C. E. Wagner, Phys. Rev. D 66 (2002) 095001.

[3] J. Cao, Z. Xiong and J. M. Yang, Phys. Rev. Lett. 88 (2002) 111802.

[4] G. C. Cho, Phys. Rev. Lett. 89 (2002) 091801.

[5] S. Baek, Phys. Lett. B 541 (2002) 161.

[6] K. Cheung and W. Y. Keung, Phys. Rev. Lett. 89 (2002) 221801.

[7] K. Cheung and W. Y. Keung, Phys. Rev. D 67 (2003) 015005.

[8] C. W. Chiang, Z. Luo and J. L. Rosner, Phys. Rev. D 67 (2003) 035008.

[9] R. Malhotra and D. A. Dicus, Phys. Rev. D 67 (2003) 097703.

[10] Z. Luo, Phys. Rev. D 67 (2003) 115007.

[11] R. Hawkings, private communication.

[12] CDF Collaboration, D. Acosta et al., arXiv:hep-ex/0302009, submitted to Physical Review Letters. 
[13] K. Hagiwara and H. Murayama, Phys. Lett. B 246 (1990) 533.

[14] A. Djouadi, M. Drees and H. Konig, Phys. Rev. D 48 (1993) 3081.

[15] G. C. Cho and K. Hagiwara, Nucl. Phys. B 574 (2000) 623.

[16] J. Erler and P. Langacker, in Particle Data Group, K. Hagiwara et al. Review of Particle Physics, Phys. Rev. D 66 (2002) 010001, p. 98.

[17] ALEPH Collaboration, A. Heister et al., arXiv:hep-ex/0305071, submitted to the European Physical Journal C.

[18] M. E. Peskin and T. Takeuchi, Phys. Rev. Lett. 65 (1990) 964; Phys. Rev. D 46 (1992) 381.

[19] M. E. Peskin and J. D. Wells, Phys. Rev. D 64 (2001) 093003; J. R. Forshaw, D. A. Ross, and B. E. White, J. High Energy Phys. 10 (2001) 007; H.-J. He, N. Polonsky, and S. Su, Phys. Rev. D 64 (2001) 053004; H.-J. He, C. T. Hill, and T. M. P. Tait, ibid. 65 (2002) 055006; J. R. Forshaw, A. Sabio Vera, and B. E. White, Univ. of Manchester Report No. MC-TH-2002-14, hep-ph/0302256 (unpublished).

[20] J. L. Rosner, Phys. Rev. D 65 (2002) 073026.

[21] E. L. Berger, J. Lee and T. M. Tait, arXiv:hep-ph/0306110. 